\title{
E-Bayesian Prediction for the Burr XII Model Based on Type-II Censored Data with Two Samples
}

\author{
Hassan M. Okasha, ${ }^{1}$ Chuanmei Wang $\mathbb{D}^{2},{ }^{2}$ and Jianhua Wang ${ }^{2}$ \\ ${ }^{1}$ Department of Statistics, Faculty of Science, King AbdulAziz University, P.O. Box 80203, Jeddah 21589, Saudi Arabia \\ ${ }^{2}$ School of Science, Wuhan University of Technology, Wuhan, 430070, China \\ Correspondence should be addressed to Chuanmei Wang; 67526018@qq.com
}

Received 9 October 2019; Revised 22 December 2019; Accepted 14 January 2020; Published 1 February 2020

Academic Editor: Giorgio Kaniadakis

Copyright (c) 2020 Hassan M. Okasha et al. This is an open access article distributed under the Creative Commons Attribution License, which permits unrestricted use, distribution, and reproduction in any medium, provided the original work is properly cited.

\begin{abstract}
Type-II censored data is an important scheme of data in lifetime studies. The purpose of this paper is to obtain E-Bayesian predictive functions which are based on observed order statistics with two samples from two parameter Burr XII model. Predictive functions are developed to derive both point prediction and interval prediction based on type-II censored data, where the median Bayesian estimation is a novel formulation to get Bayesian sample prediction, as the integral for calculating the Bayesian prediction directly does not exist. All kinds of predictions are obtained with symmetric and asymmetric loss functions. Two sample techniques are considered, and gamma conjugate prior density is assumed. Illustrative examples are provided for all the scenarios considered in this article. Both illustrative examples with real data and the Monte Carlo simulation are carried out to show the new method is acceptable. The results show that Bayesian and E-Bayesian predictions with the two kinds of loss functions have little difference for the point prediction, and E-Bayesian confidence interval (CI) with the two kinds of loss functions are almost similar and they are more accurate for the interval prediction.
\end{abstract}

\section{Introduction}

The Burr type XII distribution with two parameters was first introduced by Burr [1]. The probability density function (PDF) and cumulative distribution function (CDF) of this distribution can be, respectively, written as

$$
\begin{aligned}
& f(x ; \theta, \alpha)=\theta \alpha x^{\alpha-1}\left(1+x^{\alpha}\right)^{-(\theta+1)}, x>0, \alpha>0, \theta>0, \\
& F(x ; \theta, \alpha)=1-\left(1+x^{\alpha}\right)^{-\theta}, \quad x>0 .
\end{aligned}
$$

In the following, we shall denote it by $\operatorname{Burr}(\theta, \alpha)$, where $\theta$ is the shape parameter and $\alpha$ is the scale parameter. In fact, it is basically a Pareto (type IV) model with the scale parameter $\alpha$ set to 1 in Equation (1). The inference problems with Burr distribution have been extensively investigated in the literature, and it is extremely important in the study of biological, industrial, reliability, and life testing and quality control. In the life or quality tests or experiments, a random sample $X_{1}$, $X_{2}, \cdots, X_{n}$ of size $n$ is chosen from the distribution using CDF $F(x)$ and PDF $f(x)$ for the test or experiment, but instead of continuing until all $n$ samples have failed, the test is terminated at the time of the $r^{\text {th }}(1<r<n)$ failure. The order statistics of the data is $X_{(1)} \leq X_{(2)} \leq \cdots \leq X_{(r)}$. This kind of data is called type-II censored data. Only the smallest observed values are observed, because it takes a long time to observe all the failure of $n$ individuals in some cases, and such a censoring experiment is both time-saving and cost saving. The number of the censored samples is determined before the test or experiment. Tekindal et al. evaluated left-censored data through substitution, parametric, semiparametric, and nonparametric methods [2]. The Bayesian inference is highly recommended by scholars to study censoring data. Feroze and Aslam [3] studied Bayesian analysis of Gumbel type II distribution under censored data. Tabassum et al. [4] discussed Bayesian inference from the mixture 
of half-normal distributions under censoring. Singh et al. [5] provided "Bayesian Estimation and Prediction for Flexible Weibull Model under Type-II Censoring Scheme."

Lewis [6] proposed the use of the $\operatorname{Burr}(\theta, \alpha)$ distribution as a model in accelerated life test data representing times of breakdown of an insulating fluid. Inferences and predictions for the $\operatorname{Burr}(\theta, \alpha)$ distribution and some of its testing measures based on complete and censored samples were discussed by many authors. Evans and Ragab [7] obtained Bayes estimates of $\theta$ and the reliability function based on type-II censored samples. AL-Hussaini and Jaheen $[8,9]$ obtained Bayesian estimation for the two parameters and reliability and failure rate functions of the Burr XII distribution. Ali Mousa [10] obtained empirical Bayes estimation of the parameter $\theta$ and the reliability function based on accelerated type-II censored data. Based on complete samples, Moore and Papadopoulos [11] obtained Bayesian estimates of $\theta$ and the reliability function when the parameter $\alpha$ is assumed to be known. Ali Mousa and and Jaheen [12] obtained Bayes approximate estimates for the two parameters and reliability function of the $\operatorname{Burr}(\theta, \alpha)$ distribution based on progressive type-II censored samples. Jaheen [13] used the generalized order statistics to obtain Bayesian inference for the Burr XII model. Based on progressive samples from the $\operatorname{Burr}(\theta, \alpha)$ distribution, Soliman [14] obtained the Bayesian estimates using both the symmetric (squared error) loss function and asymmetric (LINEX, general entropy) loss functions.

The E-Bayesian method is a special Bayesian method which was developed by Han [15], and it is more and more popular now. The E-Bayesian method can be used to estimate statistical distribution parameters. Gonzalez-Lopez et al. used E-Bayesian to gain flexibility in the reliability-availability system estimation based on exponential distribution under the squared error loss function [16]. Han estimated the system failure probability with the E-Bayesian method, and the relationship of E-Bayesian estimators with three different prior distributions of hyperparameters was revealed [17]. Jaheen and Okasha [18] provided the E-Bayesian parameter and reliability estimation for the Burr type XII model based on type-II censoring. However, those literatures only discussed the E-Bayesian parameter estimation or reliability for some models and lack of prediction research for Burr type XII model with type-II censoring data.

Prediction of future events on the basis of the past and present information is a fundamental problem of statistics, arising in many contexts and producing varied solutions. As in estimation, a predictor can be either a point or an interval predictor. Parametric and nonparametric predictions have been considered in the literatures. In many practical data-analytic situations, we are interested in getting the prediction interval of the statistical distribution parameters.

Prediction has been applied in medicine, engineering, business, and other areas as well. Many authors discussed prediction problems for many distributions, references to research done and review papers on prediction, in nonparametric and parametric settings, such as Al-Hussaini and Ahmad [19, 20], Al-Hussaini and Jaheen [8, 9], Ashour and El-Wakeel [21], Dunsmore [22], Guilbaud [23], Johnson et al. [24], Nigm et al. [25, 26], Patel [27], Sindhu et al. [28], and Singh et al. [5]. For more details, one can refer to Aitchison and Dunsmore [29] and Geisser [30].

In this article, an effort has been made to find Bayesian prediction bounds for future order statistics from the twoparameter Burr XII model based on type-II censored data using the two-sample prediction technique. E-Bayesian and Bayesian predictive function approaches have been used for obtaining the estimates of the unknown parameter, and some other lifetime characteristics such as the reliability and hazard functions. Bayesian estimation has been developed under symmetric and asymmetric loss functions in Section 2. E-Bayesian predictive functions are derived based on a conjugate prior for the parameter of interest and symmetric and asymmetric loss functions in Section 3. Properties of E-Bayesian predictive functions are carried out in Section 4. Finally, comparison between the new method and the corresponding Bayes techniques is made using the Monte Carlo simulation in Section 5.

\section{Bayesian Two-Sample Predictions}

Suppose that $X_{1}, X_{2}, \cdots, X_{n}$ form a random sample from the distribution with $\operatorname{CDF} F(x)$ and $\operatorname{PDF} f(x)$. The order statistics is $X_{(1)} \leq X_{(2)} \leq \cdots \leq X_{(n)}$. Let $X_{(1)}, X_{(2)}, \cdots, X_{(n)}$ have a joint $\operatorname{CDF} \tilde{F}\left(x_{(1)}, x_{(2)}, \cdots, x_{(n)}\right)$ and $\operatorname{PDF} \tilde{f}\left(x_{(1)}, x_{(2)}, \cdots\right.$, $\left.x_{(n)}\right)$. Then to the $k$ observations $x_{\left(i_{1}\right)}, x_{\left(i_{2}\right)}, \cdots, x_{\left(i_{k}\right)}$ of $X_{\left(i_{1}\right)}$ $\leq X_{\left(i_{2}\right)} \leq \cdots \leq X_{\left(i_{k}\right)}$, we can get the joint PDF:

$$
\begin{aligned}
\tilde{f}\left(x_{\left(i_{1}\right)}, x_{(2)}, \cdots, x_{(n)} ; \theta, \alpha\right) \\
=\frac{n !}{\left(i_{1}-1\right) !\left(i_{2}-i_{1}-1\right) ! \cdots\left(i_{k}-i_{k-1}-1\right) !\left(n-i_{k}\right) !} \\
\cdot\left[F\left(x_{\left(i_{1}\right)}\right)\right]^{i_{1}-1} \cdot\left[F\left(x_{\left(i_{2}\right)}\right)-F\left(x_{\left(i_{1}\right)}\right)\right]^{i_{2}-i_{1}-1} \cdots \\
\cdot\left[F\left(x_{\left(i_{k}\right)}\right)-F\left(x_{\left(i_{k-1}\right)}\right)\right]^{i_{k}-i_{k-1}-1} \\
\cdot\left[1-F\left(x_{\left(i_{k}\right)}\right)\right]^{n-i_{k}} f\left(x_{\left(i_{1}\right)}\right) f\left(x_{\left(i_{2}\right)}\right) \cdots f\left(x_{\left(i_{k}\right)}\right) .
\end{aligned}
$$

In particular,

$$
\tilde{f}\left(x_{(1)}, x_{(2)}, \cdots, x_{(n)} ; \theta, \alpha\right)=n ! f\left(x_{(1)}\right) f\left(x_{(2)}\right) \cdots f\left(x_{(n)}\right) .
$$

Suppose that $X_{(1)} \leq X_{(2)} \leq \cdots \leq X_{(r)}$ is a type-II censored sample of size $r$ obtained from a life test on $n$ items, then the joint PDF is also the likelihood function (LF), which can be written as

$$
\begin{aligned}
& \tilde{f}\left(x_{(1)}, x_{(2)}, \cdots, x_{(r)} ; \theta, \alpha\right) \\
& \quad=\frac{n !}{(n-r) !}\left[1-F\left(x_{(r)}\right)\right]^{n-r} f\left(x_{(1)}\right) f\left(x_{(2)}\right) \cdots f\left(x_{(r)}\right) .
\end{aligned}
$$


To the Burr XII distribution with the PDF (1) and CDF (2), we can get the likelihood function (LF):

$$
\begin{aligned}
\mathrm{L}(\theta, \alpha \mid \underline{x})= & \tilde{f}\left(x_{(1)}, x_{(2)}, \cdots, x_{(r)} ; \theta, \alpha\right) \\
= & \frac{n !(\theta \alpha)^{r}}{(n-r) !}\left(1+x_{(r)}{ }^{\alpha}\right)^{-(n-r) \theta} \prod_{i=1}^{r} x_{(i)}{ }^{\alpha-1}\left(1+x_{(i)}{ }^{\alpha}\right)^{-(\theta+1)} \\
= & \frac{n !}{(n-r) !} \theta^{r} \frac{x_{(i)}{ }^{\alpha-1} \alpha^{r}}{\prod_{i=1}^{r}\left(1+x_{(i)}{ }^{\alpha}\right)}\left(1+x_{(r)}{ }^{\alpha}\right)^{-(n-r) \theta} \prod_{i=1}^{r} \\
& \cdot\left(1+x_{(i)}{ }^{\alpha}\right)^{-\theta}=\frac{n ! v}{(n-r) !} \theta^{r} e^{-T \theta}
\end{aligned}
$$

where $v=\left(\prod_{i=1}^{r} x_{(i)}^{\alpha-1} \alpha^{r}\right) /\left(\prod_{i=1}^{r}\left(1+x_{(i)}{ }^{\alpha}\right)\right), \underline{x}=\left(x_{(1)}, x_{(2)}, \cdots\right.$, $\left.x_{(r)}\right), T=\sum_{i=1}^{n} \ln \left(1+x_{(i)}{ }^{\alpha}\right)+(n-r) \ln \left(1+x_{(r)}{ }^{\alpha}\right)$.

When $\alpha$ is known, the above functions with parameters $\theta$ and $\alpha$ can be rephrased only with parameter $\theta$, and we suppose $\theta$ is a random variable. According to the Bayesian theory, we use the gamma conjugate prior density for parameter $\theta$, which can be written as

$$
g(\theta \mid c, k)=\frac{k^{c}}{\Gamma(c)} \theta^{c-1} e^{-k \theta}, \quad \theta>0,
$$

where $c>0$ and $k>0$. This prior was first used by Papadopoulos [31]. The posterior density of $\theta$ given $\underline{x}$ can be obtained from (6) and (7) as follows:

$$
q(\theta \mid \underline{x})=\eta \theta^{r+c-1} e^{-(k+T) \theta}, \quad \theta>0,
$$

where $\eta=\left((k+T)^{c+r}\right) /(\Gamma(r+c))$.

2.1. Bayesian Prediction Bounds. Assume that $X_{(r)}(r=1, \cdots$, $n)$ is the $r^{\text {th }}$ ordered observation in the same sample of size $n$ independent of the informative sample of $X_{s}^{\prime}$, and they have the same distribution. Denote $Y_{s}(s=1, \cdots, m)$ as a future independent type-II censored sample from the same population with censoring scheme $s$, and suppose that $Y_{1} \leq Y_{2} \leq$ $\cdots \leq Y_{s}$ is a type-II censored sample of size $s$ obtained from a life test on $m$ items. Our aim is to develop a method to construct a Bayesian prediction about the $s^{\text {th }}(1 \leq s \leq m)$ ordered lifetime $Y_{s}$ in a future sample of size $m$. The PDF of $Y_{s}$ is given as

$$
\begin{aligned}
h\left(y_{s} \mid \theta\right)= & \frac{m !}{(s-1) !(m-s) !}\left[F\left(y_{s}\right)\right]^{s-1}\left[1-F\left(y_{s}\right)\right]^{m-s} f\left(y_{s}\right) \\
= & \frac{m !}{(s-1) !(m-s) !}\left[1-\left(1-F\left(y_{s}\right)\right)\right]^{s-1} \\
& \cdot\left[1-F\left(y_{s}\right)\right]^{m-s} f\left(y_{s}\right) \\
= & s\left(\begin{array}{c}
m \\
s
\end{array}\right) f\left(y_{s}\right) \sum_{j=0}^{s-1}\left(\begin{array}{c}
s-1 \\
j
\end{array}\right)(-1)^{j}\left[1-F\left(y_{s}\right)\right]^{m-s+j} .
\end{aligned}
$$

Substitution of $f\left(y_{s}\right)$ and $F\left(y_{s}\right)$, given by (1) and (2), respectively, yields

$$
\begin{aligned}
h\left(y_{s} \mid \theta\right)= & s\left(\begin{array}{c}
m \\
s
\end{array}\right) \theta \alpha y_{s}^{\alpha-1}\left(1+y_{s}^{\alpha}\right)^{-(\theta+1)} \sum_{j=0}^{s-1}\left(\begin{array}{c}
s-1 \\
j
\end{array}\right) \\
& \cdot(-1)^{j}\left(1+y_{s}^{\alpha}\right)^{-(m-s+j) \theta} \\
= & s\left(\begin{array}{c}
m \\
s
\end{array}\right) \theta \frac{\alpha y_{s}^{\alpha-1}}{1+y_{s}^{\alpha}} \sum_{j=0}^{s-1}\left(\begin{array}{c}
s-1 \\
j
\end{array}\right) \\
& \cdot(-1)^{j}\left(1+y_{s}^{\alpha}\right)^{-(m-s+j+1) \theta} \\
= & \theta \tau_{s} \frac{\alpha y_{s}^{\alpha-1}}{1+y_{s}^{\alpha}} \sum_{j=0}^{s-1} \rho_{j}\left(1+y_{s}^{\alpha}\right)^{-\sigma_{j} \theta},
\end{aligned}
$$

where

$$
\tau_{s}=s\left(\begin{array}{c}
m \\
s
\end{array}\right), \rho_{j}=\left(\begin{array}{c}
s-1 \\
j
\end{array}\right)(-1)^{j}, \sigma_{j}=m-s+j+1 .
$$

The Bayes predictive PDF of $Y_{s}$ is defined as

$$
h^{*}\left(y_{s} \mid \underline{x}\right)=\int_{0}^{\infty} h\left(y_{s} \mid \theta\right) q(\theta \mid \underline{x}) d \theta .
$$

Thus, combined with $h\left(y_{s} \mid \theta\right)(10)$ and the posterior PDF $q(\theta \mid \underline{x})(8)$, one has

$$
\begin{aligned}
h^{*}\left(y_{s} \mid \underline{x}\right)= & \int_{0}^{\infty} h\left(y_{s} \mid \theta\right) q(\theta \mid \underline{x}) d \theta=\frac{(r+c) \tau_{s}}{(k+T)} \frac{\alpha y_{s}^{\alpha-1}}{1+y_{s}^{\alpha}} \sum_{j=0}^{s-1} \rho_{j} \\
& \cdot\left(1+\frac{\sigma_{j}}{k+T} \ln \left(1+y_{s}^{\alpha}\right)\right)^{-(r+c+1)}, \quad y_{s}>0 .
\end{aligned}
$$

To obtain the prediction bounds of $y_{s}$, we first need to find the predictive survival function $P\left[Y_{s}>v \mid \underline{x}\right]$. It follows from (13) that

$$
\begin{aligned}
P\left[Y_{s}>v \mid \underline{x}\right]= & \int_{v}^{\infty} h^{*}\left(y_{s} \mid \underline{x}\right) d y_{s}=\tau_{s} \sum_{j=0}^{s-1} \frac{\rho_{j}}{\sigma_{j}} \\
& \cdot\left(1+\frac{\sigma_{j}}{k+T} \ln \left(1+v^{\alpha}\right)\right)^{-(r+c)}, \quad v>0 .
\end{aligned}
$$

A two-sided $100 \delta \%$ predictive interval for $Y_{s}, 1 \leq s \leq m$ is given by $P\left[L<Y_{s}<U\right]=\delta$ and denote $L(\underline{x})$ and $U(\underline{x})$ are the confidence lower and upper limits which satisfy

$$
\left.\begin{array}{l}
P\left[Y_{s}>L(\underline{x})\right]=\frac{1+\delta}{2} \\
P\left[Y_{s}>U(\underline{x})\right]=\frac{1-\delta}{2}
\end{array}\right\} .
$$


In this case, it is also not possible to obtain the solutions analytically, and one needs a suitable simulation technique for solving these nonlinear equations. And sample fractiles are used to replace the population fractiles during the simulation process. [32],

By applying the following formula due to Lingappaiah

$$
\sum_{j=0}^{n}(-1)^{\mathrm{j}}\left(\begin{array}{c}
n \\
j
\end{array}\right)(j+m)^{-1}=\frac{n !}{\prod_{j=0}^{n}(j+m)},
$$

one can get the simulation confidence limits from (14).

\subsection{Special Cases}

Case 1. To predict the first failure time $Y_{1}$ in the sample of size $m$, we set $s=1$ in (14), so that

$$
P\left[Y_{1}>v \mid \underline{x}\right]=\left(1+\frac{m}{k+T} \ln \left(1+v^{\alpha}\right)\right)^{-(r+c)} .
$$

The case $s=1$ is of particular interest; for instance, a lower limit for the first failure in a fleet of $m$ items is called a safe warranty life or an assurance limit for the fleet.

Hence, the lower and upper $100 \delta \%$ Bayesian prediction bounds for $Y_{1}$ are given, respectively,

$$
\begin{aligned}
& L_{1}(\underline{x})=\left[\exp \left(-\frac{k+T}{m}\left[1-\left(\frac{1+\delta}{2}\right)^{-1 / r+c}\right]\right)-1\right]^{1 / \alpha}, \\
& U_{1}(\underline{x})=\left[\exp \left(-\frac{k+T}{m}\left[1-\left(\frac{1-\delta}{2}\right)^{-1 / r+c}\right]\right)-1\right]^{1 / \alpha} .
\end{aligned}
$$

Case 2. The predictive survival function of $Y_{m}$ (the last lifetime in a future sample of size $m$ ) can be obtained by setting $s=m$ in (14), yielding

$$
P\left[Y_{m}>v \mid \underline{x}\right]=\sum_{j=0}^{m-1}(-1)^{j}\left(\begin{array}{c}
m \\
j+1
\end{array}\right)\left(1+\frac{j+1}{k+T} \ln \left(1+v^{\alpha}\right)\right)^{-(r+c)} .
$$

It resulted from (15) and (19) by replacing $v$ by the lower bound $L(\underline{x})$ and the upper bound $U(\underline{x})$. With a suitable numerical technique for solving these nonlinear equations, we can get the lower and upper bounds of $Y_{m}$.

2.3. The Bayesian Predictor of $Y_{s}$. With $h^{*}\left(y_{s} \mid \underline{x}\right)$ given by (13), the two sample Bayesian predictive PDF of $Y_{s}$ under squared error loss function can be obtained as

$$
\widehat{y}_{s}^{B S}=\int_{0}^{\infty} y_{s} h^{*}\left(y_{s} \mid \underline{x}\right) d y_{s}
$$

However, this integral of (20) tends to infinity, so the integral does not exist. To solve this problem, we apply the median Bayesian estimation for the two-sample Bayes prediction of $Y_{s}$. Under the symmetric (Squared error (SE)) loss function, according to the definition of median, we can see the median $\widehat{y}_{s}^{B S}$ is the solution of the equation:

$$
\tau_{s} \sum_{j=0}^{s-1} \frac{\rho_{j}}{\sigma_{j}}\left(1+\frac{\sigma_{j}}{k+T} \ln \frac{1+v^{\alpha}}{1+y_{r}^{\alpha}}\right)^{-(r+c)}=\frac{1}{2},
$$

because

$$
\begin{aligned}
P\left[Y_{s}>v \mid \underline{x}\right] & =\int_{v}^{\infty} h^{*}\left(y_{s} \mid \underline{x}\right) d y_{s} \\
& =\tau_{s} \sum_{j=0}^{s-1} \frac{\rho_{j}}{\sigma_{j}}\left(1+\frac{\sigma_{j}}{k+T} \ln \frac{1+v^{\alpha}}{1+y_{r}^{\alpha}}\right)^{-(r+c)} .
\end{aligned}
$$

And from now on, the Bayesian estimation $\widehat{y}_{s}^{B S}$ of $y_{s}$ is the median estimator; for convenience, we use the same token $\hat{y}_{s}^{B S}$.

And the Bayes point predictor of $Y_{s}$ under asymmetric (LINEX (BL)) loss function is given by

$$
\begin{aligned}
\widehat{y}_{s}^{B L}= & -\frac{1}{a} \ln \left[\int_{0}^{\infty} e^{-a y_{s}} h^{*}\left(y_{s} \mid \underline{x}\right) d y_{s}\right] \\
= & -\frac{1}{a} \ln \left[\tau _ { s } \sum _ { j = 0 } ^ { s - 1 } \frac { \rho _ { j } } { \sigma _ { j } } \left(1-a \int_{0}^{\infty} e^{-a y_{s}}\right.\right. \\
& \left.\left.\cdot\left(1+\frac{\sigma_{j}}{k+T} \ln \left(1+y_{s}^{\alpha}\right)\right)^{-(r+c)} d y_{s}\right)\right] .
\end{aligned}
$$

\section{E-Bayesian Estimation of $\theta$}

According to Han [33], the prior parameters $c$ and $k$ should be selected to guarantee that the prior $g(\theta \mid c, k)$ in (7) is a decreasing function of $\theta$. The derivative of $g(\theta \mid c, k)$ with respect to $\theta$ is

$$
\frac{d g(\theta \mid c, k)}{d \theta}=\frac{k^{c}}{\Gamma(c)} \theta^{c-2} e^{-k \theta}[(c-1)-k \theta]
$$

Thus, for $0<c<1, k>0$, the prior $g(\theta \mid c, k)$ is a decreasing function of $\theta$.

Assuming that the hyperparameters $c$ and $k$ in (7) are independent and $\pi(c, k)=\pi_{1}(c) \pi_{2}(k)$, the E-Bayesian estimate of parameter $\theta$ (expectation of the Bayesian estimate of $\theta$ ) is

$$
\widehat{\theta}^{\mathrm{EB}}=E(\theta \mid \underline{x})=\iint_{D} \widehat{\theta}^{B}(c, k) \pi(c, k) d c d k,
$$

where $D$ is the domain of $c$ and $k$ for which the prior density is decreasing in $\theta$, and $\widehat{\theta}^{B}$ is the Bayes estimate of $\theta$. For more details, see Han [34] and Jaheen and Okasha [18].

3.1. The E-Bayesian Predictor of $Y_{s}$ with Squared Error Loss Function. E-Bayesian estimate of $\theta$ is obtained based on three different distributions of the hyperparameters $c$ and $k$. These 
TABLe 1: Prediction of Example 1 for the real data with sample size $n=15$.

\begin{tabular}{cccccccc}
\hline$s$ & BL1 & BS1 & BS1 CI & EBS1 & EBS1 CI & EBL1 & EBL1 CI \\
1 & 0.4263764 & 0.4316624 & $(0.2576238,0.5902167)$ & 0.4257137 & $(0.3786459,0.4628089)$ & 0.4174119 & $(0.3695664,0.4584008)$ \\
2 & 0.5237016 & 0.5259481 & $(0.3823931,0.6691212)$ & 0.5186851 & $(0.4608387,0.5644443)$ & 0.5142769 & $(0.4546417,0.5652961)$ \\
3 & 0.5941900 & 0.5944176 & $(0.4607947,0.7375225)$ & 0.5861901 & $(0.5200581,0.6387599)$ & 0.5837638 & $(0.5153327,0.6425961)$ \\
4 & 0.6616387 & 0.6603902 & $(0.5269148,0.8136975)$ & 0.6512217 & $(0.5765007,0.7110461)$ & 0.6503505 & $(0.5731552,0.7170255)$ \\
5 & 0.7496692 & 0.7448745 & $(0.5982316,0.9329483)$ & 0.7344847 & $(0.6474641,0.8051043)$ & 0.7360397 & $(0.6461408,0.8144799)$ \\
\hline$s$ & BL2 & BS2 & BS2 CI & EBS2 & EBS2 CI & EBL2 & EBL2 CI \\
1 & 0.4065425 & 0.410962 & $(0.2454158,0.5614648)$ & 0.4103752 & $(0.3748135,0.4511887)$ & 0.4025163 & $(0.3647253,0.4450713)$ \\
2 & 0.4985693 & 0.5006385 & $(0.3642232,0.6357492)$ & 0.4997995 & $(0.4561466,0.5500556)$ & 0.4956201 & $(0.4489861,0.5483002)$ \\
3 & 0.5652026 & 0.5654322 & $(0.4387903,0.6996264)$ & 0.5645469 & $(0.514723,0.6221456)$ & 0.5622232 & $(0.5088181,0.6227278)$ \\
4 & 0.6286810 & 0.6275569 & $(0.501555,0.7699742)$ & 0.6266803 & $(0.5705216,0.6919983)$ & 0.6257744 & $(0.5659482,0.6945529)$ \\
5 & 0.7098164 & 0.7064558 & $(0.5690331,0.8778819)$ & 0.7057107 & $(0.6406105,0.7823044)$ & 0.7069225 & $(0.6379363,0.7888539)$ \\
\hline$s$ & BL3 & BS3 & BS3 CI & EBS3 & EBS3 CI & EBL3 & EBL3 CI \\
1 & 0.4392170 & 0.4465164 & $(0.2664276,0.6111139)$ & 0.4409658 & $(0.401057,0.4644336)$ & 0.4325223 & $(0.3911746,0.4624098)$ \\
2 & 0.5399871 & 0.5442798 & $(0.3955065,0.6935378)$ & 0.5374641 & $(0.4883087,0.5664592)$ & 0.5332061 & $(0.4809603,0.5704103)$ \\
3 & 0.6130198 & 0.6154904 & $(0.4766979,0.765482)$ & 0.6077103 & $(0.5513518,0.6410906)$ & 0.6056173 & $(0.5458406,0.6482854)$ \\
4 & 0.6832925 & 0.6843914 & $(0.5452837,0.8463613)$ & 0.6756226 & $(0.6116752,0.7137244)$ & 0.6752716 & $(0.6078378,0.7231206)$ \\
5 & 0.7764433 & 0.7732495 & $(0.6194648,0.9751638)$ & 0.7630916 & $(0.6880245,0.8083315)$ & 0.7655518 & $(0.6860387,0.8221346)$ \\
\hline
\end{tabular}

distributions are used to investigate the influence of the different prior distributions on the E-Bayesian estimation of $\theta$.

The following distributions of $c$ and $k$ may be used

$$
\begin{aligned}
& \pi_{1}(c, k)=\frac{1}{b B(u, v)} c^{u-1}(1-c)^{v-1}, \quad 0<c<1,0<k<b \\
& \left.\pi_{2}(c, k)=\frac{2(b-k)}{b^{2} B(u, v)} c^{u-1}(1-c)^{v-1}, \quad 0<c<1,0<k<b\right\}, \\
& \pi_{3}(c, k)=\frac{2 k}{b^{2} B(u, v)} c^{u-1}(1-c)^{v-1}, \quad 0<c<1,0<k<b
\end{aligned}
$$

where $B(u, v)$ is the beta function. For $\pi_{i}(c, k), i=1,2,3$, the E-Bayesian estimate of $Y_{\mathrm{s}}$ with squared error loss function is obtained from (21) and (26) as

$$
\widehat{y}_{s}^{\mathrm{EBS}_{i}}=\iint_{D} \widehat{y}_{s}^{\mathrm{BS}} \pi_{i}(c, k) d k d c
$$

3.2. E-Bayesian Point Predictor of $Y_{s}$ with LINEX Loss Function. Based on the LINEX loss function, the E-Bayesian estimation of $\theta$ can be computed for the three different distributions of the hyperparameters $c$ and $k$ given by (26). For $\pi_{i}(c, k), i=1,2,3$, the E-Bayesian estimator of $Y_{\mathrm{s}}$ with LINEX loss function is obtained from (23) and (26) as below:

$$
\widehat{y}_{s}^{\mathrm{EBL}}=\iint_{D} \widehat{y}_{s}^{\mathrm{BL}} \pi_{i}(c, k) d k d c
$$

Analytical and numerical computations for the integrals in (27) and (28) are very complicated. With Monte Carlo simulation, we can get all of the estimators, and the samples are shown in part 4 .
3.3. Properties of E-Bayesian Point Predictor of $Y_{s}$. As the integral of $\hat{y}_{s}^{\mathrm{BS}}(21)$ does not exist, we cannot get the expression of real $\hat{y}_{s}^{\mathrm{BS}}$, and we can only get the median estimation of $\hat{y}_{s}^{\mathrm{BS}}$ with (22). So, we cannot prove the properties of $\mathrm{E}$ Bayesian point predictor of $Y_{s}$, but with our experience of E-Bayesian estimation [18], we can only guess the relations among $\widehat{y}_{s}^{\mathrm{EBS}_{i}}$ and $\hat{y}_{s}^{\mathrm{EBL}_{i}},(i=1,2,3)$ as follows:

$$
\begin{aligned}
& \text { (i) } \widehat{y}_{s}^{\mathrm{EBS}_{2}}<\widehat{y}_{s}^{\mathrm{EBS}_{1}}<\widehat{y}_{s}^{\mathrm{EBS}_{3}} \\
& \text { (ii) } \lim _{T \rightarrow \infty} \widehat{y}_{\mathrm{s}}^{\mathrm{EBS}_{1}}=\lim _{T \rightarrow \infty} \widehat{y}_{\mathrm{s}}^{\mathrm{EBS}_{2}}=\lim _{\mathrm{T} \rightarrow \infty} \widehat{y}_{\mathrm{s}}^{\mathrm{EBS}_{3}} \\
& \text { (iii) } \widehat{y}_{\mathrm{s}}^{\mathrm{EBL}_{2}}<\widehat{y}_{\mathrm{s}}^{\mathrm{EBL}_{1}}<\widehat{y}_{\mathrm{s}}^{\mathrm{EBL}_{3}} \\
& \text { (iv) } \lim _{T \rightarrow \infty} \widehat{y}_{s}^{\mathrm{EBL}_{1}}=\lim _{T \rightarrow \infty} \widehat{y}_{s}^{\mathrm{EBL}_{2}}=\lim _{T \rightarrow \infty} \widehat{y}_{s}^{\mathrm{EBL}_{3}}
\end{aligned}
$$

The relationship of $\hat{y}_{s}^{\mathrm{EBS}_{i}}, \widehat{y}_{s}^{\mathrm{EBL}_{i}}(i=1,2,3)$ can be observed only, and we cannot give the proof. However, the examples in part 4 can confirm this relationship.

In order to verify our parameter estimation, sample prediction, and the above relationship, the following examples are given to illustrate them.

\section{Monte Carlo Simulation and Comparisons}

4.1. Illustrative Example with Real Data. To verify the estimation and prediction method of this paper, we give two illustrative examples. A complete sample from a clinical trial describes a relief time (in hours) for 50 arthritic patients given by Wingo [35] and used recently by Ahmed et al. $[36,37]$ and $\mathrm{Wu}$ et al. [38]. Wingo [35], and Ahmed et al. $[36,37]$ showed that the Burr type XII model was acceptable for these data. Ahmed et al. [37] obtained the estimation of the parameters as $\widehat{\alpha}=5.115$ and $\widehat{\theta}=7.0651$. 


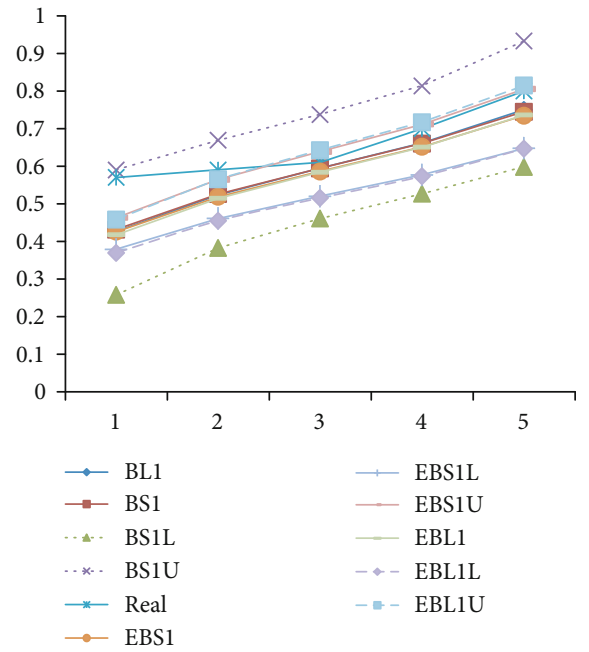

(a)

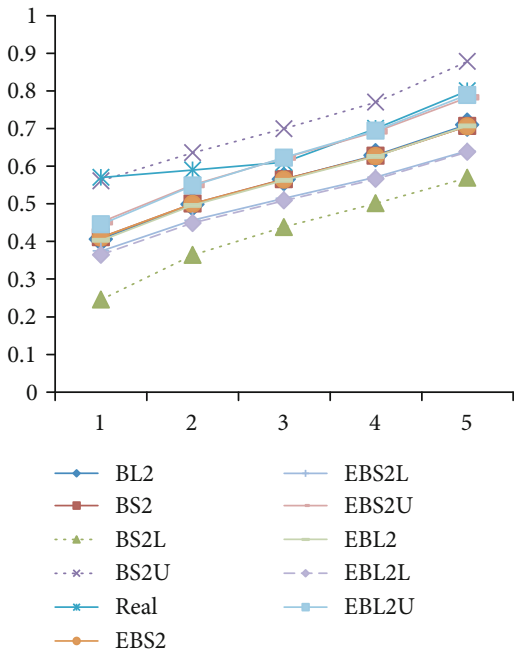

(b)

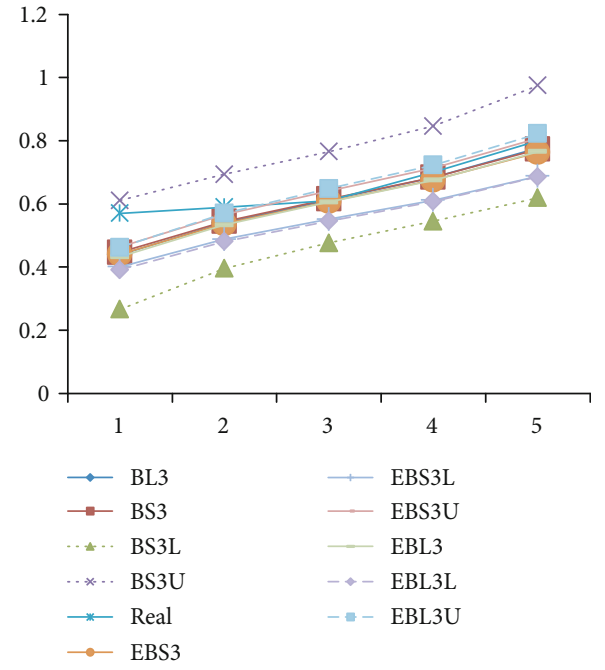

(c)

FIgURE 1: The prediction curves.

TABLe 2: Prediction of Example 2 for the real data with sample size $n=50$.

\begin{tabular}{cccccccc}
\hline$s$ & BL1 & BS1 & BS1 CI & EBS1 & EBS1 CI & EBL1 & EBL1 CI \\
1 & 0.4427966 & 0.4543341 & $(0.2722825,0.6114758)$ & 0.4538649 & $(0.4466274,0.460834)$ & 0.4441464 & $(0.42695,0.4613846)$ \\
2 & 0.5446005 & 0.5539914 & $(0.4060942,0.690365)$ & 0.5534132 & $(0.544460,0.5620414)$ & 0.5477999 & $(0.527261,0.5684298)$ \\
3 & 0.6182980 & 0.6266535 & $(0.4911443,0.7592645)$ & 0.6259903 & $(0.615667,0.6359494)$ & 0.6224449 & $(0.5998507,0.645974)$ \\
4 & 0.6890106 & 0.6970331 & $(0.5633366,0.837345)$ & 0.6962807 & $(0.6844733,0.70769)$ & 0.6943775 & $(0.6694205,0.719781)$ \\
5 & 0.7831541 & 0.7878778 & $(0.641267,0.9639144)$ & 0.7869955 & $(0.7729321,0.800626)$ & 0.7879466 & $(0.7599305,0.816337)$ \\
\hline$s$ & BL2 & BS2 & BS2 CI & EBS2 & EBS2 CI & EBL2 & EBL2 CI \\
1 & 0.4393090 & 0.4508057 & $(0.270185,0.6065865)$ & 0.4512354 & $(0.4461563,0.458294)$ & 0.4416102 & $(0.425065,0.4584106)$ \\
2 & 0.5401777 & 0.5496258 & $(0.402952,0.6846776)$ & 0.5501589 & $(0.5438776,0.558895)$ & 0.5446061 & $(0.5248414,0.564781)$ \\
3 & 0.6131909 & 0.6216188 & $(0.4873143,0.752772)$ & 0.6222362 & $(0.6149967,0.632314)$ & 0.6187336 & $(0.5970456,0.641236)$ \\
4 & 0.6831240 & 0.6912727 & $(0.558888,0.8297724)$ & 0.6919834 & $(0.6837077,0.703519)$ & 0.6901039 & $(0.666393,0.7147314)$ \\
5 & 0.7757520 & 0.7810124 & $(0.6360892,0.954093)$ & 0.7818692 & $(0.772022,0.7956296)$ & 0.7827897 & $(0.756385,0.8103091)$ \\
\hline$s$ & BL3 & BS3 & BS3 CI & EBS3 & EBS3 CI & EBL3 & EBL3 CI \\
1 & 0.4453643 & 0.4572579 & $(0.274020,0.6155324)$ & 0.4564765 & $(0.449534,0.4612051)$ & 0.4467165 & $(0.429975,0.4636027)$ \\
2 & 0.5478573 & 0.5576113 & $(0.4086974,0.695090)$ & 0.5566453 & $(0.548053,0.5625018)$ & 0.5510368 & $(0.530939,0.5708726)$ \\
3 & 0.6220617 & 0.6308316 & $(0.494319,0.7646674)$ & 0.6297189 & $(0.619806,0.6364815)$ & 0.6262050 & $(0.6040166,0.648505)$ \\
4 & 0.6933625 & 0.7018194 & $(0.5670253,0.843662)$ & 0.7005487 & $(0.689201,0.7083007)$ & 0.6987046 & $(0.674852,0.7230455)$ \\
5 & 0.7886493 & 0.7935954 & $(0.645565,0.9721487)$ & 0.7920867 & $(0.7785474,0.801358)$ & 0.7931679 & $(0.7661625,0.819795)$ \\
\hline
\end{tabular}

Example 1. The following data sample was generated from the two parameter Burr type XII distribution with $\alpha=$ 5.115.0, $\theta=7.0651$; sample size $n=15 ; 10$ observations $r=$ 10 ; 5 censoring data $m=5$; to predict the 5 censoring data with $s=1,2,3,4,5$, we set $u=v=3, b=1, a=1, \delta=0.9$. The data is the sample coming from the real data and is arranged as the order statistics, and the last 5 bold ones are censored which we predict in Table 1: 0.29, 0.35, 0.36, 0.44, $0.46,0.49,0.50,0.52,0.55,0.55, \mathbf{0 . 5 7}, \mathbf{0 . 5 9}, \mathbf{0 . 6 1}, \mathbf{0 . 7 0}, \mathbf{0 . 8 0}$.

Using this data, we can get the point prediction and bound prediction of the last 5 censored data according to the method given by this paper. With the results of Equations
(21), (23), and (27)-(28), different Bayesian and E-Bayesian estimators for $Y_{\mathrm{s}}$ and its bounds are computed and are presented in Table 1. The procedure for estimating them is as follows:

(i) For given values of the prior parameters $u=v=3$, $b=1, a=1, \delta=0.9$, we generate samples from the beta distribution $c \sim \mathrm{B}(u, v)$ and uniform priors $k \sim$ $U(0, b)$, respectively

(ii) Repeat the above step (i) 10,000 times. Using the real data above, we obtain the E-Bayes estimates of $Y_{\mathrm{s}}$ and its bounds based on the BS function and BL function by simulation 
TABLe 3: MSE of Example 1 with the estimators in Table 1.

\begin{tabular}{lcccccccccc}
\hline Estimators & BL1 & BL2 & BL3 & BS1 & BS2 & BS3 & EBS1 & EBS2 & EBS3 & EBL1 \\
MSE & 0.005856 & 0.010061 & 0.00409 & 0.005618 & 0.009844 & 0.003666 & 0.006629 & 0.00999 & 0.004274 & 0.007252 \\
\hline Estimators & EBL2 & EBL3 & $L_{\text {BS1 }}$ & $U_{\text {BS1 }}$ & $L_{\text {BS2 }}$ & $U_{\text {BS2 }}$ & $L_{\text {BS3 }}$ & $U_{\text {BS3 }}$ & $L_{\text {EBS1 }}$ & $U_{\text {EBS1 }}$ \\
MSE & 0.010683 & 0.004789 & 0.024107 & 0.002179 & 0.03017 & 0.001059 & 0.020334 & 0.00521 & 0.006387 & 0.008847 \\
\hline Estimators & $L_{\mathrm{EBS} 2}$ & $U_{\mathrm{EBS} 2}$ & $L_{\mathrm{EBS3}}$ & $U_{\mathrm{EBS3}}$ & $L_{\mathrm{EBL} 1}$ & $U_{\mathrm{EBL} 1}$ & $L_{\mathrm{EBL} 2}$ & $U_{\text {EBL2 }}$ & $L_{\text {EBL3 }}$ & $U_{\text {EBL3 }}$ \\
MSE & 0.007143 & 0.011743 & 0.0031435 & 0.0084877 & 0.0072448 & 0.0086676 & 0.0082327 & 0.0119137 & 0.0038 & 0.0078657 \\
\hline
\end{tabular}

TABLE 4: MSE of example 2 with the estimators in Table 2.

\begin{tabular}{|c|c|c|c|c|c|c|c|c|c|c|}
\hline Estimators & BL1 & BL2 & BL3 & BS1 & BS2 & BS3 & EBS1 & EBS2 & EBS3 & EBL1 \\
\hline MSE & 0.003743 & 0.0040891 & 0.0035257 & 0.0030217 & 0.0032818 & 0.0028476 & 0.0030529 & 0.003247 & 0.0028904 & 0.0035903 \\
\hline Estimators & EBL2 & EBL3 & $L_{\mathrm{BS} 1}$ & $U_{\mathrm{BS} 1}$ & $L_{\mathrm{BS} 2}$ & $U_{\mathrm{BS} 2}$ & $L_{\mathrm{BS} 3}$ & $U_{\mathrm{BS} 3}$ & $L_{\mathrm{EBS} 1}$ & $U_{\mathrm{EBS} 1}$ \\
\hline MSE & 0.003803 & 0.0034056 & 0.0175073 & 0.0042639 & 0.0182517 & 0.0034877 & 0.0169131 & 0.0049894 & 0.00296 & 0.0093169 \\
\hline Estimators & $L_{\mathrm{EBS} 2}$ & $U_{\mathrm{EBS} 2}$ & $L_{\mathrm{EBS} 3}$ & $U_{\text {EBS3 }}$ & $L_{\mathrm{EBL} 1}$ & $U_{\text {EBL1 }}$ & $L_{\mathrm{EBL} 2}$ & $U_{\mathrm{EBL} 2}$ & $L_{\text {EBL3 }}$ & $U_{\mathrm{EBL} 3}$ \\
\hline MSE & 0.0029161 & 0.0099231 & 0.003253 & 0.0092306 & 0.0026306 & 0.0082243 & 0.0025454 & 0.0088625 & 0.0028125 & 0.0078276 \\
\hline
\end{tabular}

TABle 5: Prediction example with simulation.

\begin{tabular}{cccccccc}
\hline$s$ & BL1 & BS1 & BS1 CI & EBS1 & EBS1 CI & EBL1 & EBL1 CI \\
1 & 0.0989268 & 0.0643332 & $(0.0045293,0.337259)$ & 0.0634986 & $(0.058912,0.0681691)$ & 0.0962945 & $(0.0876987,0.1050439)$ \\
2 & 0.2310097 & 0.1844959 & $(0.034875,0.7016814)$ & 0.1819962 & $(0.168183,0.1961246)$ & 0.2266603 & $(0.2078706,0.2459008)$ \\
3 & 0.4209535 & 0.3657668 & $(0.0931238,1.312666)$ & 0.360525 & $(0.3313396,0.390554)$ & 0.4154838 & $(0.3817439,0.4504281)$ \\
4 & 0.7277203 & 0.6848347 & $(0.1923819,2.69180)$ & 0.6742092 & $(0.614363,0.736322)$ & 0.7197569 & $(0.6624925,0.7785412)$ \\
5 & 1.3405130 & 1.515928 & $(0.393869,9.209026)$ & 1.488853 & $(1.332795,1.653516)$ & 1.3251390 & $(1.2276826,1.4256792)$ \\
\hline$s$ & BL2 & BS2 & BS2 CI & EBS2 & EBS2 CI & EBL2 & EBL2 CI \\
1 & 0.0955290 & 0.062083 & $(0.004375,0.3241314)$ & 0.0618931 & $(0.058546,0.0664266)$ & 0.0938743 & $(0.0866777,0.1019417)$ \\
2 & 0.2228465 & 0.1777076 & $(0.0336715,0.671249)$ & 0.1771502 & $(0.167088,0.1908336)$ & 0.2207915 & $(0.2057193,0.2390398)$ \\
3 & 0.4056461 & 0.3513917 & $(0.089825,1.247777)$ & 0.3502552 & $(0.329043,0.3792505)$ & 0.4044791 & $(0.3782247,0.4372029)$ \\
4 & 0.7009810 & 0.6552626 & $(0.1852814,2.531697)$ & 0.6530585 & $(0.6097058,0.712764)$ & 0.7006364 & $(0.6570353,0.7567613)$ \\
5 & 1.2939372 & 1.438354 & $(0.3782452,8.434845)$ & 1.433241 & $(1.320916,1.590156)$ & 1.2919972 & $(1.2178218,1.3893391)$ \\
\hline$s$ & BL3 & BS3 & BS3 CI & EBS3 & EBS3 CI & EBL3 & EBL3 CI \\
1 & 0.1014997 & 0.0660396 & $(0.004646,0.347282)$ & 0.0651254 & $(0.0606048,0.068627)$ & 0.0987469 & $(0.0906665,0.1063018)$ \\
2 & 0.2371997 & 0.1896604 & $(0.035787,0.725086)$ & 0.1869064 & $(0.173261,0.1975176)$ & 0.2326070 & $(0.2146817,0.2486330)$ \\
3 & 0.4325663 & 0.3767499 & $(0.095628,1.363028)$ & 0.3709303 & $(0.3420138,0.393537)$ & 0.4266335 & $(0.3940571,0.4542965)$ \\
4 & 0.7479744 & 0.7075708 & $(0.197785,2.817802)$ & 0.6956375 & $(0.6360885,0.742565)$ & 0.7391301 & $(0.6833638,0.7852044)$ \\
5 & 1.3756176 & 1.576287 & $(0.4058139,9.83703)$ & 1.545187 & $(1.388652,1.670446)$ & 1.3587232 & $(1.2634259,1.4365982)$ \\
\hline
\end{tabular}

(iii) The computational results are summarized in Table 1 , where $\mathrm{BL}_{i}$ is the Bayesian prediction, and $\mathrm{EBL}_{i}$ is the E-Bayesian prediction, for $Y_{\mathrm{s}}$ under LINEX error loss function with three different distributions of the hyperparameters $(i=1,2,3)$. $\mathrm{BS}_{i}$ is the Bayesian estimators $\left(\mathrm{EBS}_{i}\right.$ is the E-Bayesian estimators) for $Y_{\mathrm{s}}$ under squared error loss function with three different distributions of the hyperparameters $(i=1,2,3)$. BS CI corresponds to the $90 \%$ Bayesian confidence interval, and $\mathrm{EBS}_{i} \mathrm{CI}$ corresponds to the 90\% E-Bayesian confidence interval under squared error loss function. $\mathrm{EBL}_{i} \mathrm{CI}$ corresponds to the
90\% E-Bayesian confidence interval under LINEX error loss function

Figure 1 shows us the prediction curves. Here, BSiL $(i=1,2,3)$ is the lower bound of the corresponding confidence interval, and $\mathrm{BSiU}(i=1,2,3)$ is the upper bound of the corresponding confidence interval. EBSiL $(i=1,2,3)$ and $\operatorname{EBSiU}(i=1,2,3)$ have the same meaning. These three graphs have similar results: $\mathrm{BS} i \mathrm{CI}(i=1,2,3)$ totally covers the real data, but they do not have much of degree of confidence. Especially the lower bound of BSi $(i=1,2,3)$ is far from the real data curve; EBS $i$ CI and EBL $i$ CI $(i=1,2,3)$ 


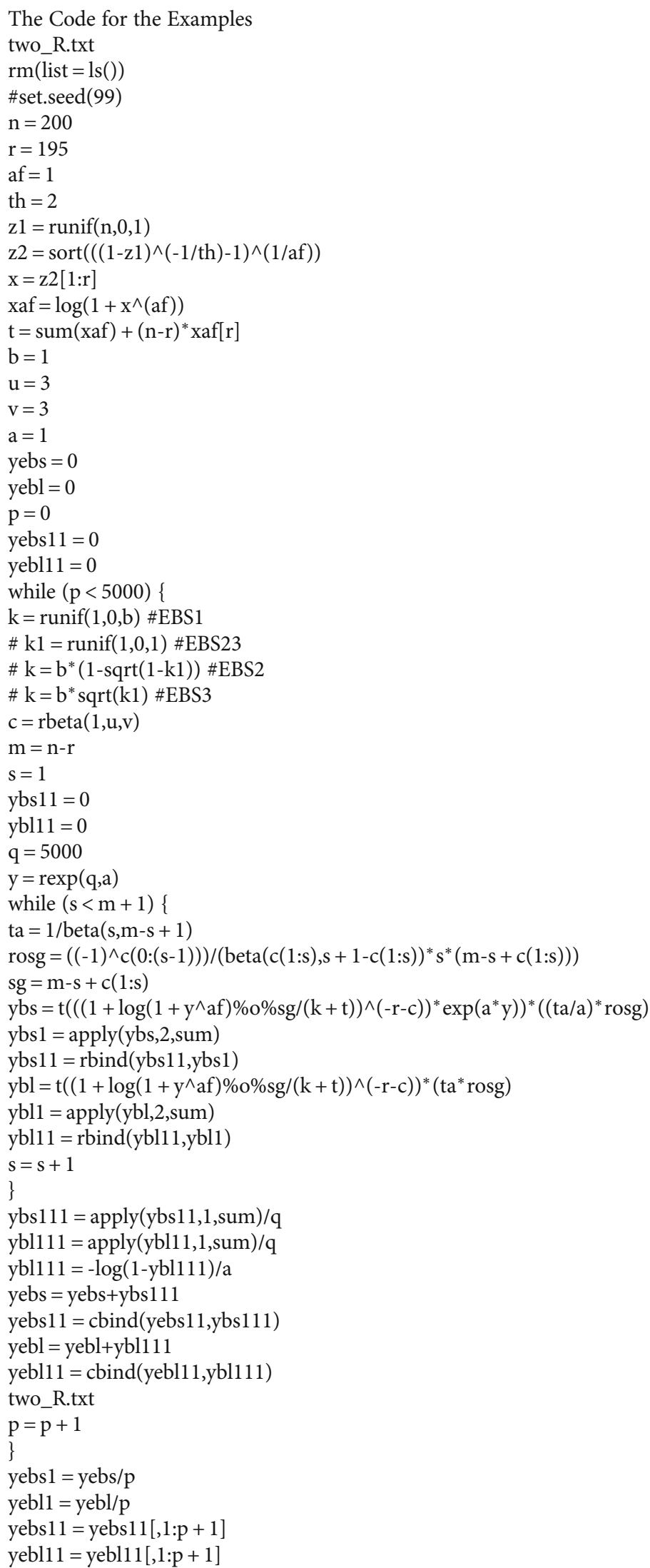




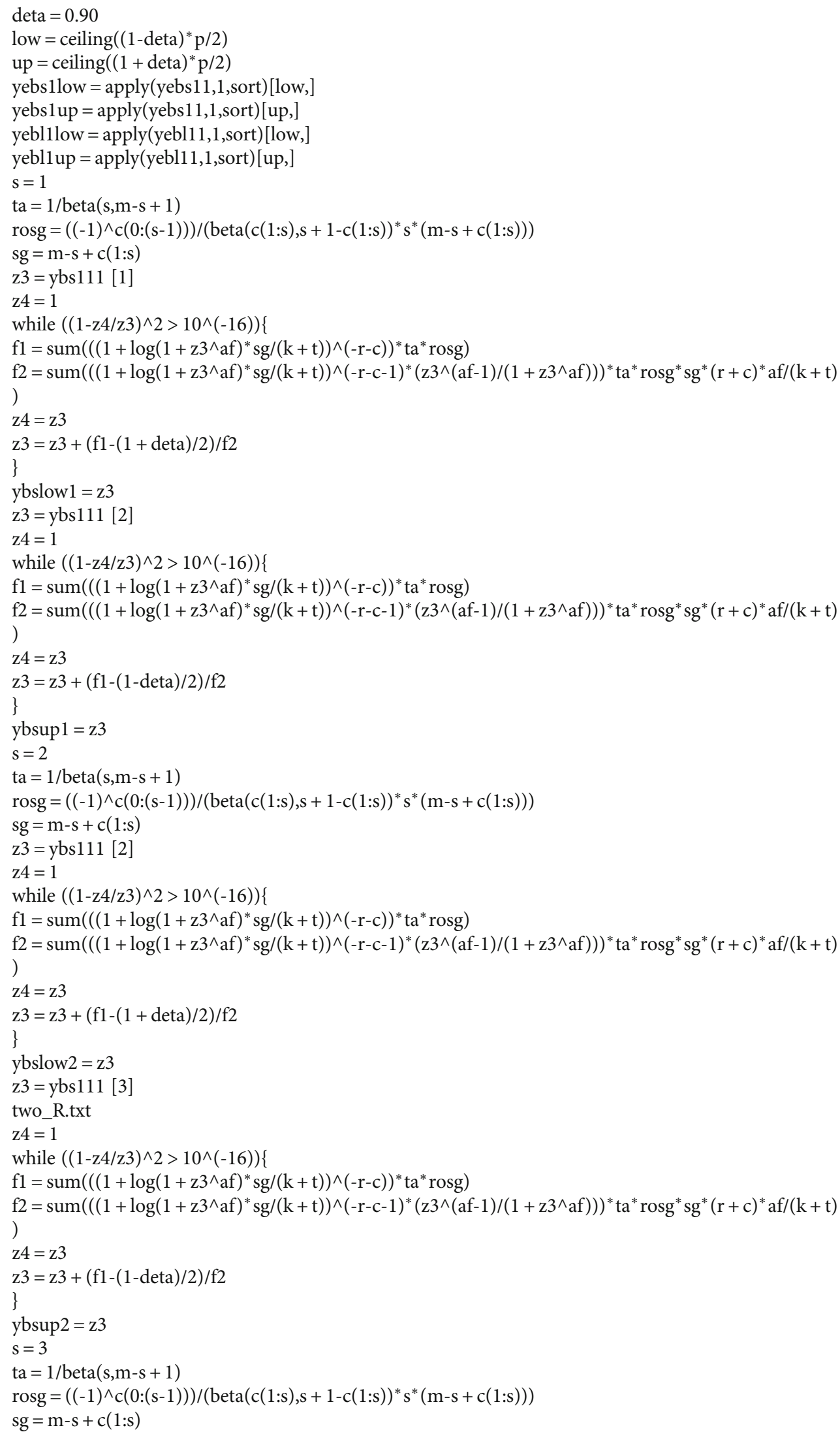

Algorithm 1: Continued. 


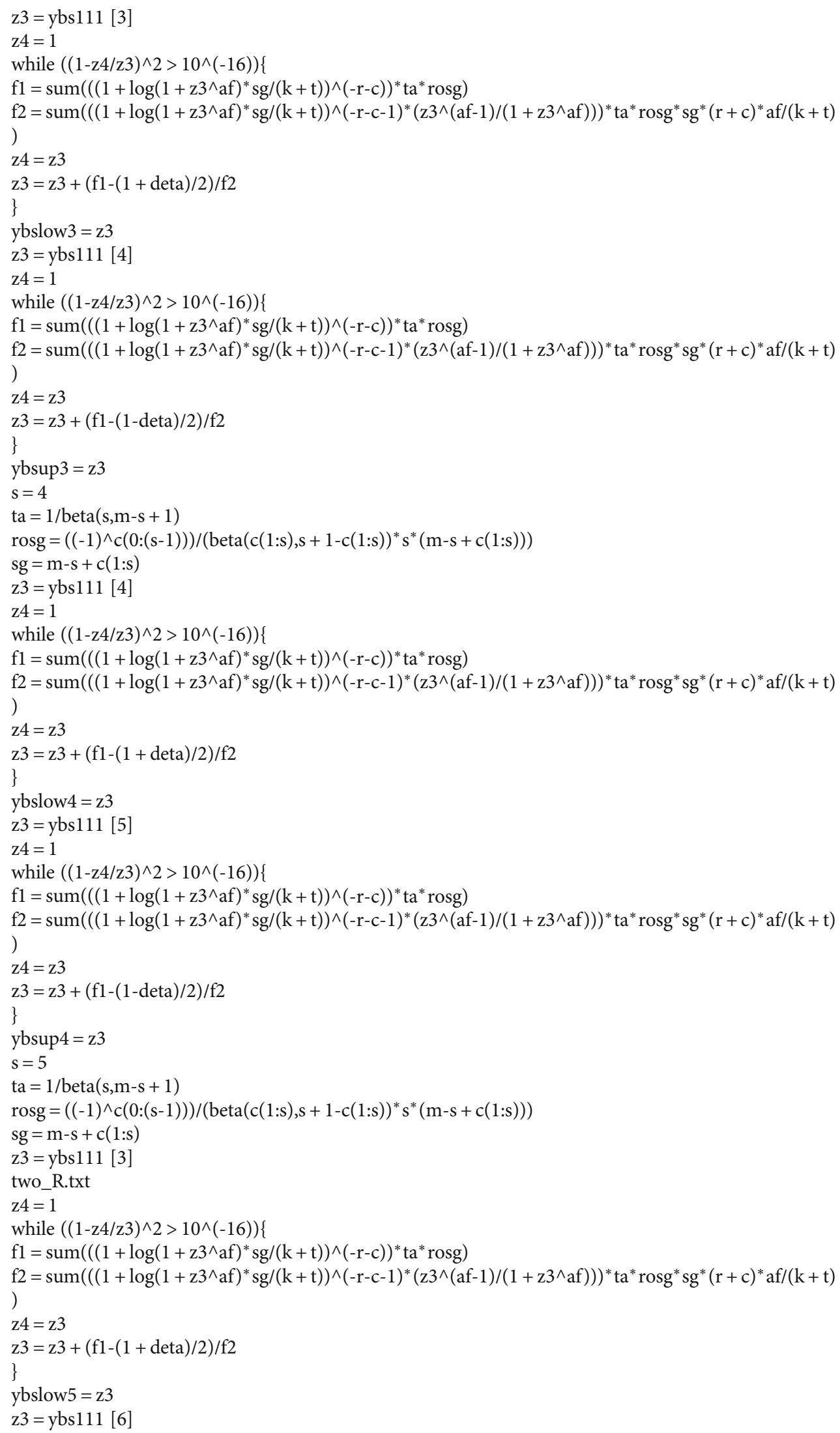




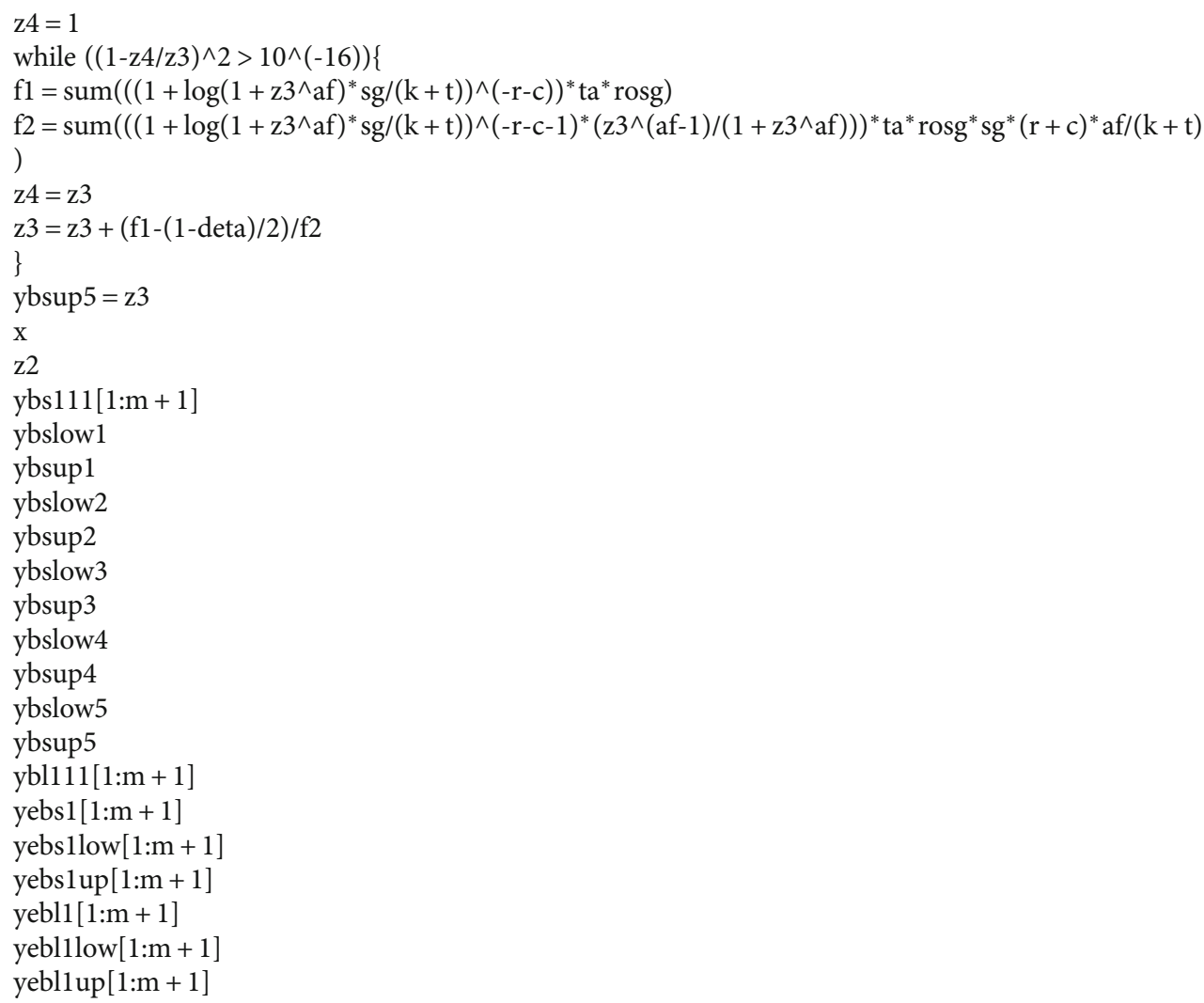

Algorithm 1

almost coincide, and their upper bounds are closest to the real data; $\mathrm{BL} i, \mathrm{BS} i, \mathrm{EBL} i$, and $\mathrm{EBS} i(i=1,2,3)$ almost coincide. So we can see that the point prediction BLi, BSi, EBLi, and $\operatorname{EBSi}(i=1,2,3)$ are not very different, the interval prediction EBSi CI and EBL $i$ CI $(i=1,2,3)$ are almost similar, and the E-Bayesian interval predictions are more accurate. The next two examples have the similar results, so we will not show the figures again.

Example 2. Another example is with the data sample generated from the same distribution as the above Example 1, but here, we enlarge the sample size to $n=50$; the meaning of other indexes is the same as the above example. Here, $r=45 ; m=5 ; s=1,2,3,4,5 ; u=v=3 ; b=1 ; a=1 ; \delta=0.9$. We get the prediction of the last 5 bold ones as Table 2 . The data are as follows:

$0.29,0.29,0.34,0.34,0.35,0.36,0.36,0.36,0.44,0.44$, $0.46,0.46,0.49,0.49,0.50,0.50,0.52,0.54,0.55,0.55,0.55$, $0.56,0.57,0.58,0.59,0.59,0.60,0.60,0.61,0.61,0.62,0.64$, $0.68,0.70,0.70,0.71,0.71,0.71,0.72,0.73, \mathbf{0 . 7 5}, \mathbf{0 . 7 5}, \mathbf{0 . 8 0}$, $0.80,0.81$.

To compare the estimators, the mean square error (MSE) is used to measure the estimation accuracy as follows. Here, to compare the bounds of the estimators, the $90 \%$ confidence limits of real data is used to calculate the MSE of our estimated bounds. For the convenience of the comparison, the MSE of Tables 1 and 2 is computed and put together as Tables 3 and 4 .
4.2. Illustrative Example with Simulation. To illustrate the operability of the methods put forward by this paper, we also give an example with simulation. The following data sample was generated from the two parameter Burr type XII distribution with $\alpha=1.0, \theta=2.0$, sample size $n=20,15$ observations $r=15,5$ censoring data $m=5$; to predict the 5 censored data with $s=1,2,3,4,5$, we set $u=v=3, b=1$, $a=1, \delta=0.9$. The data are listed as follows:

$0.05280128, \quad 0.05560962, \quad 0.06225709, \quad 0.10117384$, $0.10626390, \quad 0.11375182, \quad 0.19062614, \quad 0.24794003$, $0.24840441, \quad 0.42198706, \quad \mathbf{0 . 4 6 6 4 6 1 0 3}, \quad \mathbf{0 . 4 8 8 7 5 7 0 2}$, $0.55176242,0.65954615,0.66710844$.

\section{Conclusion}

In this paper, the E-Bayes point prediction and prediction bounds for ordered lifetime in a future sample are discussed under symmetric and asymmetric loss functions. Two examples with real data and different choices of sample size $n$ were illustrated to examine the performance of the different predictions. Comparing Tables 3 and 4, we can find with the increasing of sample size, the MSE of the Bayes predictions and E-Bayes prediction decreases, and the predictions vary with different loss functions.

A simulation study was conducted to show the feasibility of the E-Bayes prediction given in this paper. All the predictions computed with different loss functions, different sample size, and different choice of model parameters of 
the censored scheme are shown in Tables 1, 2, and 5, and the predictions with different loss functions are consistent with the relationship we put forward in 3.3. From the results, we can draw the following conclusion:

(i) From Tables 1, 2, and 5, the estimators of the E-Bayes estimates of $\hat{y}_{s}^{\mathrm{EBS}_{i}}$ and $\hat{y}_{\mathrm{s}}^{\mathrm{ELL}_{i}}(i=1,2,3)$

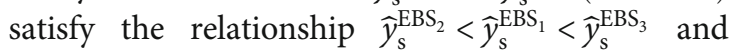
$\hat{y}_{\mathrm{s}}^{\mathrm{EBL}}<\hat{y}_{\mathrm{s}}^{\mathrm{EBL}} \mathrm{L}_{1}<\hat{y}_{\mathrm{s}}^{\mathrm{EBL}}$, and the E-Bayes estimates of different priors are more close to each other. And they are consistent with the assumption in 3.3

(ii) If one uses the E-Bayesian approach for prediction, one would expect these estimators to be better (in the sense of MSE's) than the Bayesian approach. With our examples, this cannot be seen in the results for different choices of loss functions. However, the results are similar. Because the E-Bayesian method uses more information than the ordinary Bayesian approach, it is much more reliable

(iii) When the sample size $n$ increases, the MSE of EBayesian estimators decrease. So increasing the sample size can get exacter results

(iv) The results establish that for optimum decisionmaking, importance should be given on the choice of loss function and not just the choice of prior distribution only

Burr distribution has extreme importance in the study of biological, industrial, reliability, and life testing and quality control. With the sample prediction functions, we can understand the trends and control them in a time.

\section{Data Availability}

The (DATA.doc) data used to support the findings of this study are included within Reference [36].

\section{Conflicts of Interest}

The authors declare that they have no conflicts of interest.

\section{Acknowledgments}

The authors thank the referees for their helpful remarks that improved the original manuscript. This work was financially supported by the Fundamental Research Funds for the Central Universities (WUT: 2019IA004, 2018IB016).

\section{Supplementary Materials}

The data for Example 1 and Example 2 to get the parameters' initial estimators in this manuscript are from Reference [36], and it is illustrated in the manuscript. (Supplementary Materials)

\section{References}

[1] I. W. Burr, "Cumulative frequency functions," The Annals of Mathematical Statistics, vol. 13, no. 2, pp. 215-232, 1942.

[2] M. A. Tekindal, B. D. Erdoğan, and Y. Yavuz, "Evaluating leftcensored data through substitution, parametric, semi-parametric, and nonparametric methods: a simulation study," Interdisciplinary Sciences: Computational Life Sciences, vol. 9, no. 2, pp. 153-172, 2017.

[3] N. Feroze and M. Aslam, "Bayesian analysis of gumbel type II distribution under censored data," 2014, https://www.lappublishing.com/.

[4] T. N. Sindhu, H. M. R. Khan, Z. Hussain, and B. al-Zahrani, "Bayesian inference from the mixture of half-normal distributions under censoring," Journal of the National Science Foundation of Sri Lanka, vol. 46, no. 4, pp. 587-600, 2018.

[5] S. K. Singh, U. Singh, and V. K. Sharma, "Bayesian estimation and prediction for flexible Weibull model under Type-II censoring scheme," Journal of Probability and Statistics, vol. 2013, Article ID 146140, 16 pages, 2013.

[6] A. W. Lewis, "The Burr distribution as a general parametric family in survivorship and reliability theory applications," [Ph.D. thesis], Department of Biostatistics, University of North Carolina, 1981.

[7] I. G. Evans and A. S. Ragab, "Bayesian inferences given a type2censored sample from a Burr distribution," Communications in Statistics - Theory and Methods, vol. 12, no. 13, pp. 1569$1580,1983$.

[8] E. K. Al-Hussaini and Z. F. Jaheen, "Bayesian estimation of the parameters, reliability and failure rate functions of the Burr type XII failure model," Journal of Statistical Computation and Simulation, vol. 41, no. 1-2, pp. 31-40, 1992.

[9] E. K. Al-Hussaini and Z. F. Jaheen, "Approximate Bayes estimators applied to the Burr model," Communications in Statistics - Theory and Methods, vol. 23, no. 1, pp. 99-121, 1994.

[10] M. A. M. A. Mousa, "Empirical Bayes estimators for the Burr type XII accelerated life testing model based on Type-2 censored data," Journal of Statistical Computation and Simulation, vol. 52, no. 2, pp. 95-103, 1995.

[11] D. Moore and A. S. Papadopoulos, "The Burr type XII distribution as a failure model under various loss functions," Microelectronics Reliability, vol. 40, no. 12, pp. 2117-2122, 2000.

[12] M. A. M. Ali Mousa and Z. F. Jaheen, "Statistical inference for the Burr model based on progressively censored data," Computers \& Mathematics with Applications, vol. 43, no. 10-11, pp. 1441-1449, 2002.

[13] Z. F. Jaheen, "Estimation based on generalized order statistics from the Burr model," Communications in Statistics-Theory and Methods, vol. 34, no. 4, pp. 785-794, 2005.

[14] A. A. Soliman, "Estimation of parameters of life from progressively censored data using Burr-XII model," IEEE Transactions on Reliability, vol. 54, no. 1, pp. 34-42, 2005.

[15] M. Han, "E-Bayesian method to estimate failure rate," in The Sixth International Symposium on Operations Research and Its Applications (ISORA'06), pp. 299-311, Xinjiang, China, August 2006

[16] V. A. Gonzalez-Lopez, R. Gholizadeh, and C. E. Galarza, "EBayesian estimation for system reliability and availability analysis based on exponential distribution," Communications in 
Statistics - Simulation and Computation, vol. 46, no. 8, pp. 6221-6241, 2017.

[17] M. Han, "The E-Bayesian and hierarchical Bayesian estimations for the system reliability parameter," Communications in Statistics - Theory and Methods, vol. 46, no. 4, pp. 16061620, 2017.

[18] Z. F. Jaheen and H. M. Okasha, "E-Bayesian estimation for the Burr type XII model based on type-2 censoring," Applied Mathematical Modelling, vol. 35, no. 10, pp. 4730-4737, 2011.

[19] E. K. AL-Hussaini and A. E. L.-B. A. Ahmad, "On Bayesian predictive distributions of generalized order statistics," Metrika, vol. 57, no. 2, pp. 165-176, 2003.

[20] E. K. Al-Hussaini and A. E.-B. A. Ahmad, "On Bayesian interval prediction of future records," Test, vol. 12, no. 1, pp. 79-99, 2003.

[21] S. K. Ashour and M. A. M. H. El-Wakeel, "Bayesian prediction of the median of the Burr distribution with fixed and random sample sizes," Statistics, vol. 25, no. 2, pp. 113-122, 1994.

[22] I. R. Dunsmore, "The Bayesian predictive distribution in life testing models," Technometrics, vol. 16, no. 3, pp. 455-460, 1974.

[23] O. Guilbaud, "Exact non-parametric confidence, prediction and tolerance intervals with progressive type-II censoring," Scandinavian Journal of Statistics, vol. 31, no. 2, pp. 265-281, 2004.

[24] R. A. Johnson, J. W. Evans, and D. W. Green, "Nonparametric Bayesian predictive distributions for future order statistics," Statistics \& Probability Letters, vol. 41, no. 3, pp. 247-254, 1999.

[25] A. M. Nigm, "Prediction bounds for the Burr model," Communications in Statistics - Theory and Methods, vol. 17, no. 1, pp. 287-297, 1988.

[26] A. M. Nigm, E. K. Al-Hussaini, and Z. F. Jaheen, "Bayesian two-sample prediction under the Lomax model with fixed and random sample size," Journal of Applied Statistical Science, vol. 15, pp. 381-390, 2007.

[27] J. K. Patel, "Prediction intervals - a review," Communications in Statistics - Theory and Methods, vol. 18, no. 7, pp. 23932465, 1989.

[28] T. N. Sindhu, H. M. R. Khan, Z. Hussain, and T. Lenzmeier, "Bayesian prediction from the inverse rayleigh distribution based on type-II trim censoring," Journal of Statistics and Management Systems, vol. 20, no. 5, pp. 995-1008, 2017.

[29] J. Aitchison and I. R. Dunsmore, "Statistical prediction analysis," Bulletin of the American Mathematical Society, vol. 82, no. 5, pp. 683-688, 1976.

[30] S. Geisser, Predictive Inference. An Introduction, Chapman and Hall, London, 1993.

[31] A. S. Papadopoulos, "The Burr distribution as a failure model from a Bayesian approach," IEEE Transactions on Reliability, vol. R-27, no. 5, pp. 369-371, 1978.

[32] G. S. Lingappaiah, "Sequential life-testing with spacings, exponential model," IEEE Transactions on Reliability, vol. R-30, no. 4, pp. 370-374, 1981.

[33] M. Han, "E-Bayesian estimation and hierarchical Bayesian estimation of failure rate," Applied Mathematical Modelling, vol. 33, no. 4, pp. 1915-1922, 2009.

[34] M. Han, "E-Bayesian estimation of the reliability derived from Binomial distribution," Applied Mathematical Modelling, vol. 35 , no. 5, pp. 2419-2424, 2011.
[35] D. R. Wingo, "Maximum likelihood methods for fitting the burr type XII distribution to life test data," Biometrical Journal, vol. 25, no. 1, pp. 77-84, 1983.

[36] A. A. Soliman, A. H. A. Ellah, N. A. Abou-Elheggag, and A. A. Modhesh, "Bayesian inference and prediction of Burr type XII distribution for progressive first failure censored sampling," Intelligent Information Management, vol. 3, no. 5, pp. 175185, 2011.

[37] A. A. Soliman, A. H. Abd Ellah, N. A. Abou-Elheggag, and A. A. Modhesh, "Estimation from Burr type XII distribution using progressive first-failure censored data," Journal of Statistical Computation and Simulation, vol. 83, no. 12, pp. 22702290, 2013.

[38] S.-F. Wu, C. C. Wu, Y. L. Chen, Y. R. Yu, and Y. P. Lin, "Interval estimation of a two-parameter Burr-XII distribution under progressive censoring," Statistics, vol. 44, no. 1, pp. 77-88, 2010. 